\title{
Massive star population in circumnuclear star-forming regions
}

\author{
Mar Álvarez-Álvarez, Ángeles I. Díaz, and Marcelo Castellanos \\ Departamento de Física Teórica, C-XI, Universidad Autónoma de \\ Madrid, Cantoblanco, E-28049 Madrid, España
}

\begin{abstract}
Due to their high luminosity, the importance of understanding the massive star formation and evolution of giant $\mathrm{H}$ II regions has become more and more evident in the last few years. A mayor scenario where giant $\mathrm{H}$ II regions form and develop are the very inner parts of some galaxies. These bursts frequently are arranged in a ring-like pattern. We present a study of the stellar populations and gas physical conditions in circumnuclear star-forming regions (CNSFR) based on broad- and narrow-band photometry and spectrophotometric data, which have been analyzed with the use of evolutionary population synthesis and photo-ionization models. It is found that most CNSFRs show composite stellar populations of slightly different ages. They seem to have the highest abundances found in $\mathrm{H}$ II region-like objects, showing also N/O overabundances and S/O underabundaces by a factor of about three. Also, CNSFRs as a class segregate from the disk H II region family, clustering around higher ionizing temperatures.
\end{abstract}

\section{Observations and data sample}

\subsection{Photometric data}

Two data sets of broad and narrow band photometry were obtained for 20 nearby galaxies. The filters used were $U, B, R, I, \mathrm{H} \alpha$ and $\mathrm{H} \beta$. The observed galaxies are bright $(14.1>B>8.96 \mathrm{mag})$ and nearby $(8.4<d<65 \mathrm{Mpc})$ spirals of different morphological and nuclear type. A total of 332 circumnucelar star-forming regions (CNSFRs) in the sample galaxies have been measured and analyzed. The observed colors have been corrected for reddening using the observed $\mathrm{H} \alpha / \mathrm{H} \beta$ ratio.

\subsection{Spectrophotometric data}

Data of moderate resolution $(1.4-2.5 \AA / \mathrm{pix})$ and wide coverage $(3500-9700 \AA)$ were obtained with the WHT telescope (ORM). These configuration allows the observation of both oxygen ([O II] and [O III]) and sulphur ([S II] and [S III]) emission lines for the diagnostics of the emitting gas.

\section{Analysis of broad-band colors: stellar population synthesis models}

The broad-band colors have been analyzed in combination with the $\mathrm{H} \alpha$ equivalent widths obtained through narrow filters, with the use of the stellar population 
synthesis models by Leitherer et al. (1999). Single bursts of subsolar, solar and twice solar metallicities have been tested. Only a small fraction of the observed objects, the bluest and the reddest, can be reproduced by the models. Continuous star formation provides even worse fits. The great majority of the regions can be explained only by the combination of at least two populations of different ages. In order to explore this possibility, we have constructed models of two populations of the same metallicity and slightly different ages according to two parameters: $t(\mathrm{Myr})$, which represents the difference in age of the two assumed populations, and $f$, which represents the ratio of the mass of the youngest population to the total mass of the star cluster. These models reproduce quite well the $W_{H \alpha^{-}}(R-I)$ relation, but the situation is more complicated when the $(U-B)$ colors are considered in the analysis. Not even the composite population models are able to reproduce the location of the HII regions in the $(U-B)-(R-I)$ diagram, with half of the regions showing $(U-B)$ colors much redder than any model predicition. This fact needs to be studied further both from the observational and theoretical points of view, in order to understand how star formation takes place in these $\mathrm{H}$ II regions.

\section{Analysis of emission line spectra: photo-ionization models}

Diagnostic diagrams have been used in order to check whether CNSFRs present different fundamental parameters in comparison to high-metallicity disk $\mathrm{HII}$ regions. The CNSFRs sample includes regions in NGC 3310 and NGC 7714, known to have subsolar oxygen abundances, and those high metallicity regions in NGC 1068, NGC 2903, NGC 3351, NGC 3504 and NGC 5953. As a comparison sample, we have selected high metallicity disk H II regions from Díaz \& PérezMontero (2000) and Bresolin et al. (1999). CNSFRs segregate from the disk H II regions in the $\log \left(\left[\mathrm{O}_{\mathrm{II}}\right] /[\mathrm{O} \mathrm{III}]\right)$ vs. $\log \left([\mathrm{S} \mathrm{II}] /\left[\mathrm{S} \mathrm{III}^{\mathrm{II}}\right]\right)$ diagram. The former, cluster around the value of $\log \eta^{\prime}=0.0$ while the latter do around $\log \eta^{\prime}=0.8$. This parameter is a measure of the hardness of the ionizing radiation and seems to imply higher ionizing temperatures for the CNSFRs. Regarding relative abundances, it seems that the high metallicity CNSFRs present an overabundance in nitrogen in comparison to disk $\mathrm{H}$ II regions, when these regions are plotted in the $\log ([\mathrm{N} \mathrm{II}] /[\mathrm{O} \mathrm{II}])$ vs. $\log ([\mathrm{OII}] /[\mathrm{O} \mathrm{III}])$ diagram. In order to reproduce the location of CNSFRs in the $\operatorname{logS23}$ versus $\log ([\mathrm{S} \mathrm{II}] /[\mathrm{S} \mathrm{III}])$ diagram, a reduction of the S/O abundance by a factor of about three must be invoked, which does not affect neither the $[\mathrm{N} \mathrm{II}] /[\mathrm{O} \mathrm{II}]$ nor $[\mathrm{O} \mathrm{II}] /[\mathrm{O} \mathrm{III}]$ line-intensity ratios.

\section{References}

Bresolin, F., Kennicutt, R.C., Garnett, D.R. 1999, ApJ 510, 104

Díaz, A.I., Pérez-Montero, E. 2000, MNRAS 312, 130

Leitherer, C., Schaerer, D., Goldader, J.D., et al. 1999, ApJS 123, 3 